\title{
3D Numerical Simulation of Flow and Mixing Performances in Novel Tri-screw and Four-screw Extruders
}

\author{
Chongxin Wang ${ }^{\mathrm{a}, *}$, Xiangzhe Zhu ${ }^{\mathrm{b}}$ \\ School of Mechanical Engineering, Liaoning Shihua University, Fushun, China \\ awangcx11@sina.com, bxzzhu@126.com \\ *Corresponding author
}

Keywords: tri-screw extruder; four-screw extruder; mesh superposition technique; mixing efficiency

\begin{abstract}
Screw extruder is an important equipment for mixing processing. This paper briefly introduces the physical models of novel tri-screw and four-screw extruders. Using the software Polyflow, the three-dimensional transient finite element models are employed to explore the complex flow and mixing characteristics of polymer fluids in tri-screw and four-screw extruders. The mixing index of particles distributed in the special center regions of tri-screw and four-screw extruders are analyzed specially. Moreover, the statistical post-processing, software of Polystat, is used to calculate the mixing index probability distribution, time-averaged mixing efficiency, instantaneous time mixing efficiency, logarithmic stretching rate, shear rate and axial distance mixing index. Parameters mentioned before between the four-screw, twin-screw and tri-screw extruders are compared in order to better understand the mixing mechanism of the novel four screw extruder. The results show that the mixing efficiency of the four-screw extruder is better than that of the twin-screw extruder and the tri-screw extruder.
\end{abstract}

\section{Introduction}

With the development of the polymer processing industry, as one of the main equipment for polymer processing, screw extruder has been widely used. Researchers have done a lot of research on this equipment from experiments and numerical simulations [1]. The comprehensive properties of the final blend were analyzed, and the mixing performance of the screw extruder was indirectly evaluated [2]. Using numerical simulation method, flow field action and other evaluation indicators, the flow field action and distribution of each area of the equipment, analyzed the central area especially[3].

In recent years, the multi-screw extruder has been developed. Multi-screw extruder technology, with its unique advantages, makes up for the shortcomings of single and twin-screw extruders, and has begun to receive more and more attention in the field of extrusion. Multi-screw extruder mainly includes tri-screw extruder and four-screw extruder. The research on tri-screw is more common, but the research on four-screw extruder is less. In this paper, the effects of different screw combinations on the mixing effect of screw extruders were analyzed by statistical analysis of time-averaged mixing efficiency, instantaneous time mixing efficiency, mixing index, logarithmic stretching and shear rate[4-5]. Taking the screw extruder to engage the conventional conveying components as the research object, the flow of the polymer melt in the four-screw extruder is analyzed. It provides a theoretical basis for the optimal design of multi-screw extruder.

\section{Theoretical model}

\subsection{Physical model}

In this paper, the finite element model of the screw adopts the tetrahedral division method, and the finite element model of the flow channel adopts the hexahedron division method. In order to more accurately observe the flow field change between the central area of the screw extruder and 
the screw and the barrel, further fine-grain the center area of the flow path. Using four layers of boundary layer division, the thickness of the boundary layer is the thickness of the screw gap to ensure a higher quality mesh, capturing more subtle changes in the flow field. Finally, the finite element model of the screw and flow field is overlapped and combined by mesh superposition technique (MST) of Polyflow software. The dimensions of the screw extruder are as follows: the barrel radius is $17.5 \mathrm{~cm}$; the screw root radius is $13 \mathrm{~cm}$; the screw tip radius screw is $17 \mathrm{~cm}$; the centerline distance of screws is $31.5 \mathrm{~cm}$; the total length of the screw is $60 \mathrm{~cm}$, and the number of screw edges is 3 . The finite element models of the twin-screw, tri-screw and four-screw extruder contain 222152, 333228 and 420304 cells, respectively.
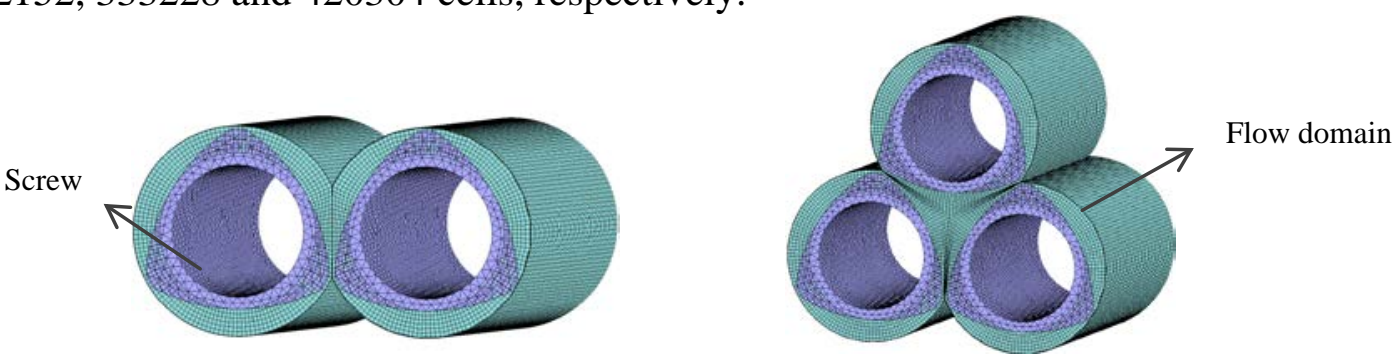

Figure 1 Finite element model of screw extruder

Figure 1 shows the finite element (FE) model of the twin-screw extruder and the tri-screw extruder. The screw rotates counterclockwise in the same direction. Figure 2(a) shows the geometry of the four-screw extruder. The screw rotates counterclockwise in the same direction. The construction of rhombic-layout four screw extruder is between the tri-screw extruder with the screw-triangular distribution and the square-layout four screw extruder. It has the geometric characteristics of them. Figure 2(b) and (c) show the finite element model of the four screw extruder. With the periodic rotation of the screw, the central region of the two triangles in the basin exhibits corresponding periodic changes.

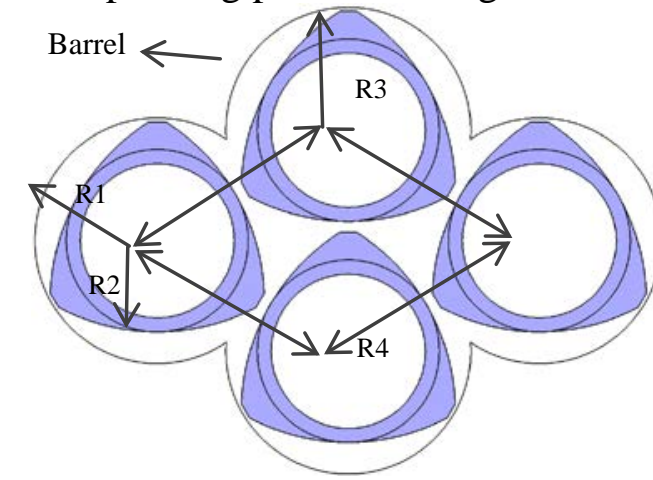

(a) Geometric model

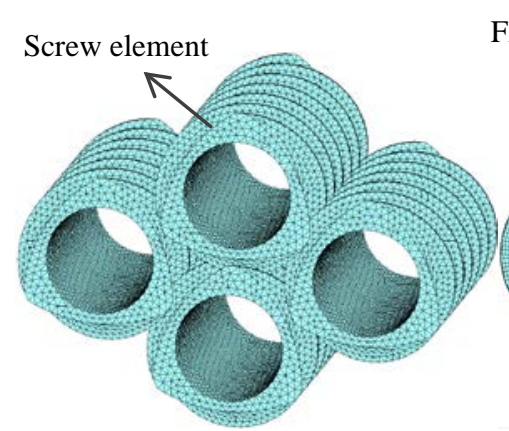

(c) FE model of flow domain

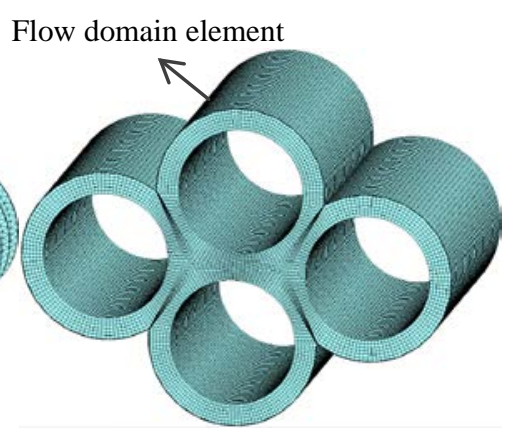

(b) FE model of screw

of the four-screw extruder

\subsection{Simulated conditions}

The screw extruder simulation boundary conditions are set as follows:

(1) The flow inlet sets the inflow rate to $10 \times 10^{-6} \mathrm{~m}^{3} / \mathrm{s}$;

(2) The inner surface of the barrel has no slip according to the basic assumption, setting the normal speed and the tangential speed to zero, $V_{\mathrm{n}}=0, V_{\mathrm{s}}=0$;

(3) Screw rotating speed is $60 \mathrm{r} / \mathrm{min}$;

(4) The flow outlet sets the normal force and the tangential force to zero, $F_{\mathrm{n}}=0, F_{\mathrm{s}}=0$.

Because the transient viscosity model Bird-Carreau has a low power factor index, it is nonlinear. Therefore, in the calculations, the velocity and coordinates are quadratic interpolation, the pressure is normal pressure, and the viscosity adopts slowly (Picard iterative method). The Krank-Nikkorson method is used to calculate the transient flow field (Crank-Nicolson Method). In order to reduce the influence of the Weissenberg phenomenon and the axial velocity, the screw rotating speed takes 60 $\mathrm{r} / \mathrm{min}$. The convergence accuracy level of all simulations is $1 \times 10^{-5}$. 


\section{Results and discussions}

Figure 3 shows the mixing index distribution of radial section of three combined screw extruders. The magnitude of fluid mixing index between 0.5 and 1 is the most in the three kinds of extruders. It indicates that the material particles experience the stronger tensile action, and the stretching flow is more favorable for the material mixing in the extruders. In addition, there is a flowing dead zone near the screw wall in the three kinds of extruders, so it shows that a blue area in the mixing index distribution. Among the three kinds of extruders, the four-screw extruder has the highest peak value of 0.809 . Figure 3 (a), (b) and (c) show the mixing index distribution in the axial section $\mathrm{z}=10 \mathrm{~cm}$ of the three kinds of extruders. Figure 3(d), (e) and (f) show the mixing index distribution of the axial section $\mathrm{z}=32 \mathrm{~cm}$ in the three kinds of extruders. It can be seen that the mixing index of the three extruders is close to orange in the area near the screw edge, and the four-screw extruder is red near the screw edge. It shows the mixing index is higher in four-scew exturder, the stretching effect of material is better in this area. Figure 3(c) and (f) show that the four-screw extruder has two meshing zones, and the material is flowing frequently in the two meshing zones, and the mixing index is higher than the tri-screw extruder, because the tri-screw extruder has the single meshing zone. In conclusion, the four-screw extruder has better mixing effect, less dead zone of the material flowing than the other models. The materials exchange frequently between meshing areas.

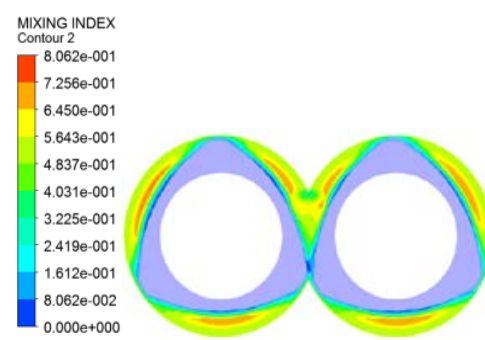

(a) $\mathrm{z}=10 \mathrm{~cm}$

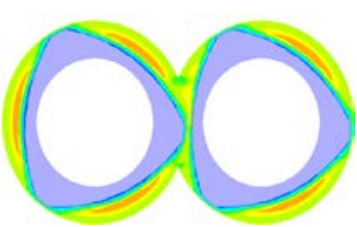

(d) $\mathrm{z}=32 \mathrm{~cm}$

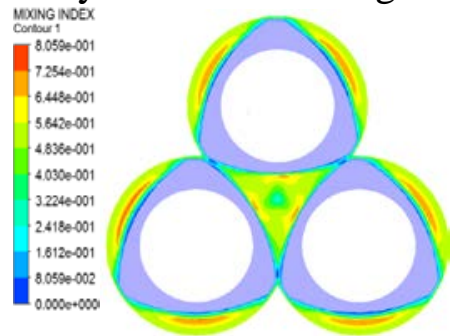

(b) $\mathrm{z}=10 \mathrm{~cm}$

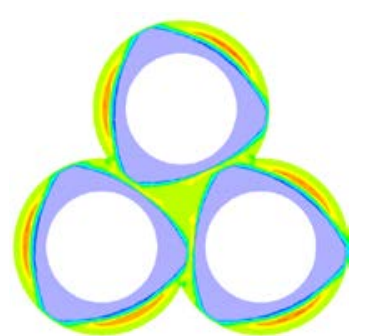

(e) $\mathrm{z}=32 \mathrm{~cm}$

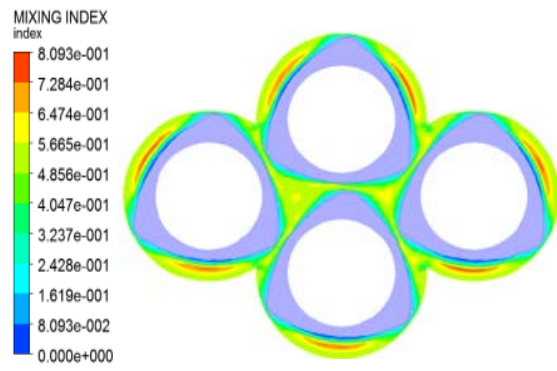

(b) $\mathrm{z}=10 \mathrm{~cm}$

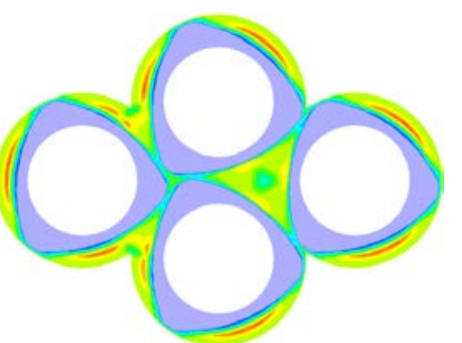

(f) $\mathrm{z}=32 \mathrm{~cm}$

Figure 3 Mixing index distributions of radial sections

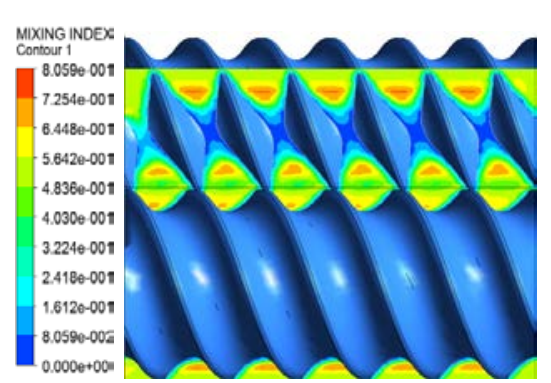

(a)Tri-screw extruder

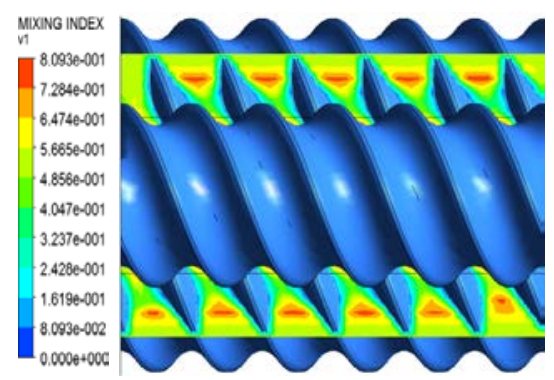

(b)Four-screw extruder

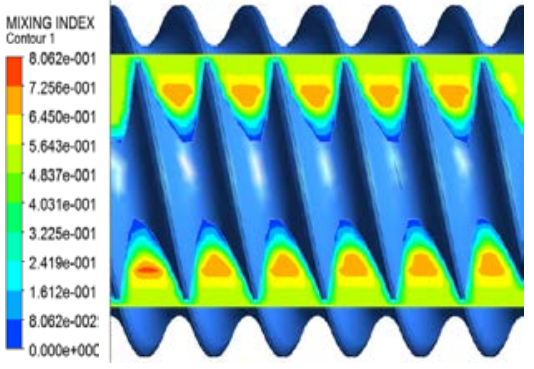

(c)Twin-screw extruder

Figure 4 Mixing index distributions of different axial sections

Figure 4 shows the axial mixing index of three combined screw extruders. Figure 4(a), (b), and (c) show the axial mixing index distributions at cross-section of the tri-screw extruder (a), four-screw extruder (b) and twin-screw extruder (c). On the whole, the mixing index of the three models tends to be stable at the positions in the z-axis direction, and the mixing effect at each position is similar, the mixing effect is similar in each position. In Figure 4(a), it can be seen that the area close to the wall surface of the screw is blue, indicating a lower mixing index. It is difficult to see a blue area at the 
cross section of the four-screw extruder, and the four-screw extruder has a large red area, and the red areas in other models are small. It shows that the phenomenons of shearing and stretching flow in the four-screw extruder mixing process is obvious.

By comparing the average of instantaneous mixing efficiency in three models during the time increasing, it is found that the average of instantaneous mixing efficiency increases sharply at the initial stage under the all models, and then fluctuates gradually within a certain range with mixing time. Due to the special geometric configuration, the particle is in a complex state of stretching (the average of instantaneous mixing efficiency is positive) and compressing (the average of instantaneous mixing efficiency is negative). As shown in Figure 5(a), the average of instantaneous mixing efficiency fluctuates aperiodically. It can be seen that the instantaneous mixing efficiency of the four-screw extruder is significantly higher than the other two kinds of extruders when the fluctuation is at the beginning to be stable (10 s to $40 \mathrm{~s}$ interval). After 40 seconds, the instantaneous mixing efficiency of each extruder tends to be stable, and the value of mixing efficiency is close to that other two models, it shows that the four-screw extruder is more efficient than the tri-screw extruder and the twin-screw extruder.

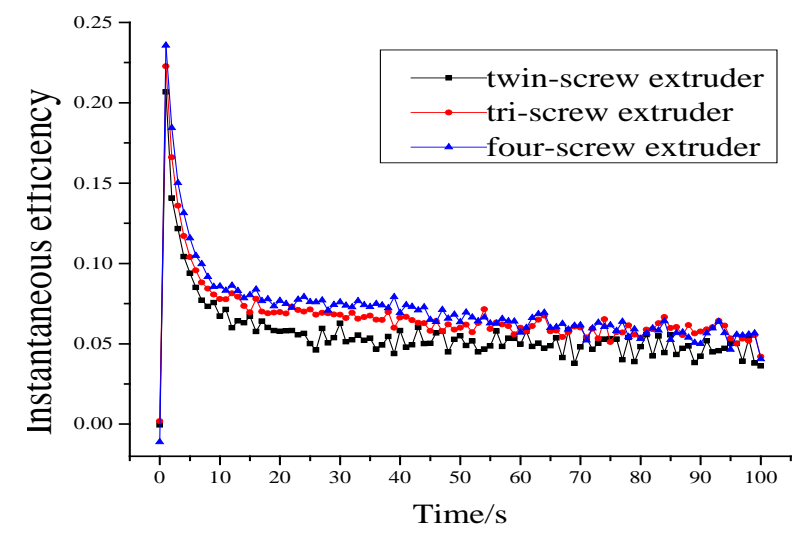

(a) Instantaneous mixing efficiency

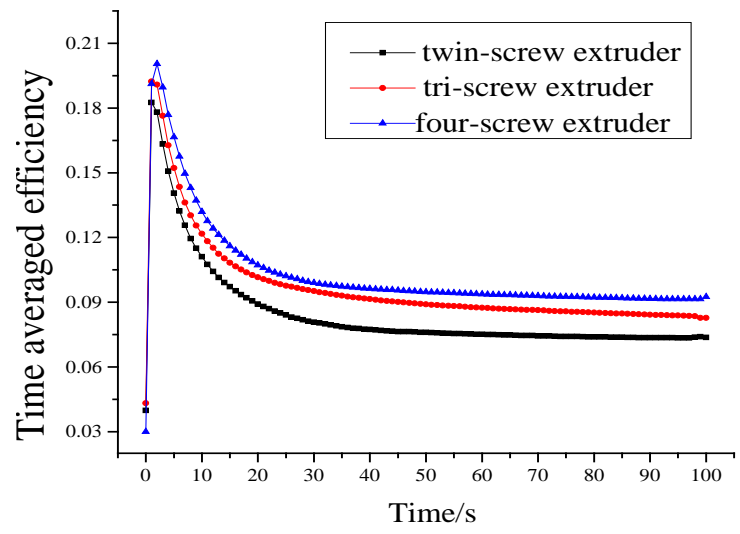

(b) Averaged time mixing efficiency

Figure 5 Comparision of instantaneous mixing efficiency and averaged time mixing efficiency between three models

As shown in Figure 5(b),it shows that the averaged time mixing efficiency value is higher, the mixing efficiency is better of the screw extruders. By comparison curve of the time averaged mixing efficiency values of different screw combination extruders is shown in the figure. It can be seen that the time averaged mixing efficiency value of the screw extruder under various combinations at the initial moment increases sharply. With the mixing time increasing, the time averaged mixing efficiency value is tends to be gentle, which is a necessary condition for effective mixing of the screw extruder. It is known from the figure that as time increases, the time averaged mixing efficiency value gradually approaches stability. It can be seen that the time averaged mixing efficiency of the four-screw is greater than the values of the tri-screw and the twin-screw. After the smoothing, the time averaged mixing efficiency of the four-screw is also greater than the other two combinations. The time averaged mixing efficiency of the tri-screw is greater than the mixing efficiency of the twin-screw, indicating that the four-screw extruder is more efficient than the triscrew extruder and twin-screw extruder.

Many literatures have shown that tensile flow is more effective than shear flow in mixing efficiency, so logarithmic stretching is an important date for comparing mixing efficiency. In order to compare the logarithmic stretching of three kinds of different extruders, 5000 material points were uniformly released at the end of each flow at the initial time, these material points neither interact nor affect the flowing field. As shown in Figure 6(a), it can be seen from this figure that with the increase of time, the stretching rate of screw extruder increases exponentially, this is a characteristic of chaotic mixing. As shown in the figure, the logarithmic stretching of the four-screw extruder and tri-screw extruder is significantly greater than that of the twin-screw extruder and the logarithmic stretching of four-screw extruder is slightly larger than the tri-screw extruder. It is shown that the 
four-screw extruder has higher mixing efficiency, so the mixing efficiency of four-screw extruder is better than the others.

Figure 6(b) shows the shear rate distribution curves of the three kinds of models during the axial length direction. It can be seen that the shear rate increases with the increase of the axial distance, when the axial distance is $15 \mathrm{~cm}$ to $55 \mathrm{~cm}$, the shear rate changes obviously. The shear rate of the four-screw extruder is higher than the other two kinds of models. It indicates that the four-screw extruder has the better mixing efficiency in mixing process.

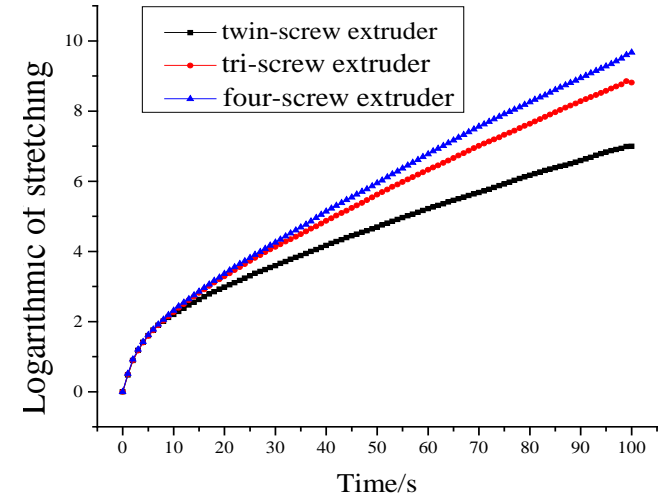

(a) Logarithmic stretching

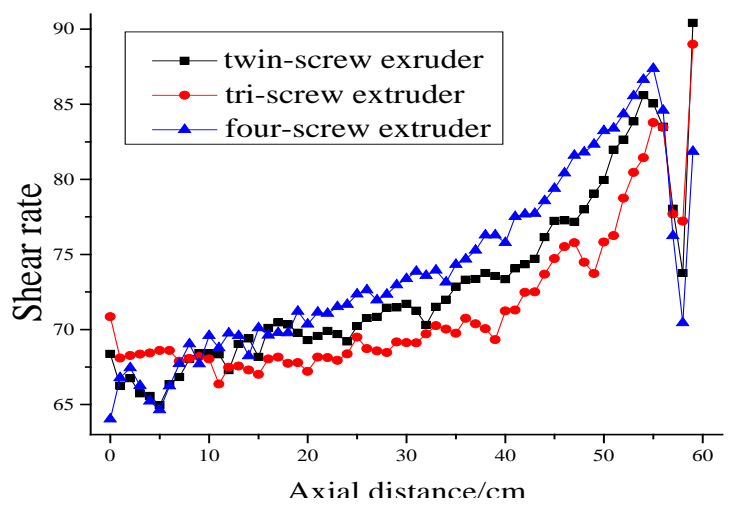

(b) Shear rate

Figure 6 Comparisions of logarithmic stretching and shear rate between three models

Figure 7(a) shows the mixing index probability distribution curve of the three kinds of extruders. As you can see from the figure, when the values of the three curves are between 0 and 0.5 , the red, black and blue curves shift to the right way. When the mixing index is greater than 0.5 , the black, red and blue curves shift to the right way. The mixing index is between 0 and 0.5 , the flow form is shear flow, the mixing index is greater than 0.5 , and the flow form is tensile flow, while the contribution of the polymer in extensional flow is higher than the mixing shear flow. In the shear flow process, the mixing efficiency of the twin-screw extruder is higher than the other two combinations. In the stretching process that contributes more to the mixing effect, three combinations have similar mixing efficiency. It can be seen that the efficiency of the twin-screw and four-screw extruders is slightly higher than the mixing efficiency of the tri-screw extruder. Three curves appear black, red, blue in turn shifted slightly to the right, indicating that the shear and extensional flow sequentially slightly increased over time.

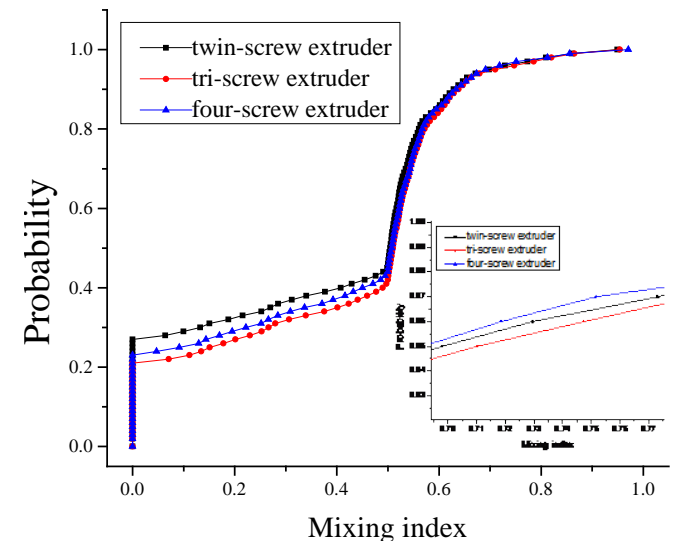

(a) Mixing index

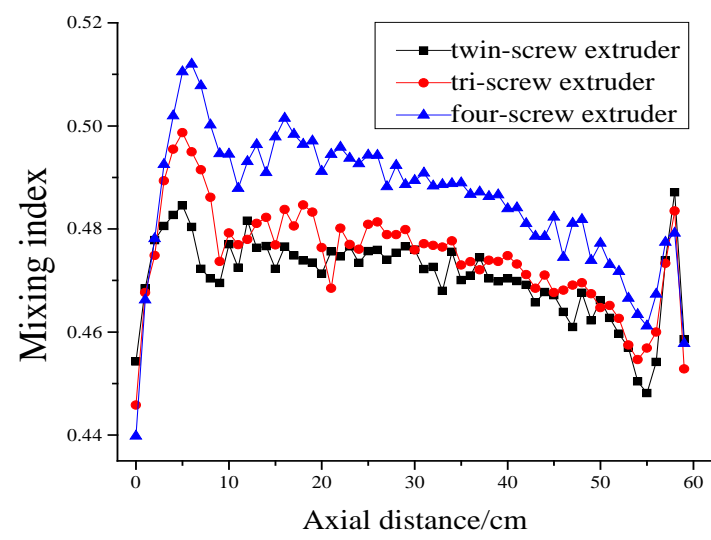

(b) Axial distance of mixing index

Figure 7 Comparisions of mixing index probability and axial distance of mixing index between three models

As shown in Figure 7(b), the distribution curve of the of the three combined screw extruders in the axial distance. At the axial distance of 0 to $5 \mathrm{~cm}$, the mixing index of the three combined models rises sharply, showing a relatively fast upward trend. The peak of the mixing index of the four-screw extruder is much higher than the other two combined models. The tri-screw extruder is also higher than the mixing index of the twin-screw extruder. At the axial distance of 5 to $55 \mathrm{~cm}$, the mixing 
index of the three combinations tends to decrease slowly, and the mixing index of the four-screw extruder is higher than the other two combinations. In the final stage, the mixing index of the three combinations has an upward trend, reaching a similar mixed index. It shows that the process of mixing and stretching of the four-screw extruder is more complete and the effect is better than the other two combinations.

\section{Conclusion}

From the distribution of the mixing index, the four-screw extruder has two meshing center zones, and the material stretching frequently in the two meshing zones, which is higher than the mixing index of the three-screw extruder in the single meshing zone. The material in the four-screw extruder has a more obvious shear-stretching flow between the screws, and the four-screw extruder has a small dead zone, it has a higher dispersion mixing efficiency than the others. From the figure, the time-averaged mixing efficiency and instantaneous time mixing efficiency of the four-screw extruder are the largest in the three models, and the fluctuation trend is stable. It can be seen from the axial distance mixing index that the mixing index of the four-screw extruder is higher than that of the twin-screw extruder and the tri-screw extruder. Showing the mixing effect at each position, the fourscrew extruder has a big advantage. From the shear rate distribution curve, the shear rate of the three models is increasing gradually. The shear rate of the four-screw extruder is slightly larger than the other three combinations. In summary, the four-screw extruder has the best mixing effect.

\section{Acknowledgements}

This work was supported by National Natural Science Foundation of China (grant No. 51473073, 50903042 and 51303075); Program for Liaoning Excellent Talents in University (grant No. LR2016030); Natural Science Foundation of Liaoning Province (Grant no. 2015020142).

\section{References}

[1] Jiang, N., Zhu, C.W. (2001) Analysis of the mixing effect of three-screw extruder. China Plastics, 15, 87-90.

[2] Zhu, X.Z., Yuan, H.Q., Wang, W.Q. (2009) Numerical simulation of flow characteristics in new co-rotating triangle arrayed triple screw extruders. Journal of Materials Processing Technology, 209, 89-99.

[3] Zhu, X.Z, Xie,Y.J, Yuan, H.Q. (2008) Numerical simulation of etrusion characteristics of intermeshing co-rotating tri-screw extruder. Polym. Mater. Sci. Eng., 24, 32-35.

[4] Rauwendsslc. (2003) Elongational mixing in foam extrusion.Cellular Polymers, 22, 103-115.

[5] Connelly, R.K., Kokini, J.L. (2003) 2D numerical simulation of differential viscoelastic fluids in a single-screw continuous mixer: Application of viscoelastic finite element methods. Adv. Polym.Tech., 22, 22-41. 